\title{
Microwave and Interferometer Diagnostics prepared for first Plasma Operation of WENDELSTEIN 7-X
}

\author{
M. Hirsch ${ }^{1}$,
}

E-mail: matthias.hirscheipp.mpg.de

\author{
A. Benndorf ${ }^{1}$, E. Blanco ${ }^{2}$, R. Burhenn ${ }^{1}$, A. Cappa ${ }^{2}$, T. Estrada ${ }^{2}$, K. Ewert ${ }^{1}$, \\ G. Gliege ${ }^{1}$, H.-J. Hartfuss ${ }^{1}$, C. Heuer ${ }^{1}$, R. Herrmann ${ }^{1}$, W. Kasparek ${ }^{3}$, \\ J. P. Knauer ${ }^{1}$, P. Kornejew ${ }^{1}$, A. Krämer-Flecken ${ }^{4}$, F. Lapayese ${ }^{2}$, \\ B. van Milligen ${ }^{2}$, S. Mohr ${ }^{1}$, U. Neuner ${ }^{1}$, L. Pacios ${ }^{2}$, A. de la Peña ${ }^{2}$, \\ D. Pilopp ${ }^{1}$, B. Plaum ${ }^{4}$, N. Rüter ${ }^{1}$, T. Sieber ${ }^{1}$, T. Stange ${ }^{1}$, M. Steffen ${ }^{1}$, T. \\ Sunn-Pedersen ${ }^{1}$, H. Trimino-Mora ${ }^{1}$, D. Wagner ${ }^{1}$, T. Windisch ${ }^{1}$ and S. Wolf ${ }^{4}$ \\ ${ }^{1}$ Max-Planck Institut für Plasmaphysik, Greifswald/Garching, Germany \\ ${ }^{2}$ Centro de Investigationes, Medioambientales y Technologicas (CIEMAT), Madrid, Spain \\ ${ }^{3}$ Inst. für Grenzflächenverfahrenstechnik und Plasmatechnologie, Univ. Stuttgart, Germany \\ ${ }^{4}$ Institut für Energie- und Klimaforschung, Forschungszentrum Jülich, Germany
}

For the first test operation of W7-X, only a limited set of seven diagnostics is considered to be mandatory. Among these are the Dispersion Interferometer for the electron density measurement and the ECE radiometer. Dispersion Interferometry using a $10.6 \mu \mathrm{m} \mathrm{CO}_{2}$-laser and its second harmonic with no need for a reference line is widely independent of mechanical vibrations and drifts and thus developed as a candidate for steady state density control. The 32-channel ECE radiometer probing the electron temperature profile using Gaussian optics and a single broadband mixer is dedicated to track ECRH heating and perform first transport studies. Three reflectometers are being prepared to use the first plasmas for their test operation namely a Doppler reflectometer with an optimized Gauss telescope optics, a Doppler reflectometer with a fast steering antenna with no movable in-vessel parts capable for a fast scan of the turbulence Kspectrum, and a conventional correlation reflectometer probing the separatrix region.

First EPS Conference on Plasma Diagnostics - $1^{\text {st }}$ ECPD

14-17 April 2015,

Villa Mondragone, Frascati (Rome) Italy

\section{Introduction}

For the superconducting stellarator Wendelstein 7-X (W7-X) at Greifswald, Germany [1], currently in its commissioning phase a pulse length of $30 \mathrm{~min}$ is planned at $8 \mathrm{MW}$ ECR heating embedding high-power phases with 10s additional NBI of up to $10 \mathrm{MW}$. The first test operation phase, referred to as OP1.1 and scheduled late summer 2015 will have uncooled wall elements and instead of the later island divertor a plasma edge defined by 5 inertially cooled graphite inboard limiters to protect the already installed in-vessel components [2]. The integral heat input for this configuration is restricted to $<2 \mathrm{MJ}$ for which more then $5 \mathrm{MW}$ ECR heating are 
available. Physics exploration of W7-X is planned to start about a year later with a fully cooled first wall and inertially cooled island divertor which allows about 10s operation at $8 \mathrm{MW}$ ECRH for example. Full 30 min steady state discharges are subject of OP2 scheduled to start 2019 with the geometrically identical but water cooled cw-divertor.

Diagnostic development for W7-X [3] is oriented along the planned physics program [4] with the particular challenges of long pulse operation: The expected $\mathrm{cw}$ heat load at diagnostic frontends is around $100 \mathrm{~kW} / \mathrm{m}^{2}$ which requires active cooling. Steady state ECR heating at 140 $\mathrm{GHz}$ results in a level of several ten $\mathrm{kW} / \mathrm{m}^{2}$ of nearly isotropic microwave stray radiation in the torus in particular during high-density operation, where the absorption of the ECRH beams is moderate only [5].

For microwave diagnostics $\mathrm{W} 7-\mathrm{X}$, with a large major radius $R=5.5 \mathrm{~m}$ and rather large aspect ratio $R / a \sim 10$, offers good conditions with well separated cyclotron resonances and the option of probing locations where the curvature of the flux surfaces is moderate only. Microwave diagnostics using in-vessel stainless steel optics and $\mathrm{Cu}$ waveguide components the latter only if direct plasma radiation can be avoided - provide the possibility for operation with high heat- and particle loads even under steady-state and reactor relevant conditions. Sensitive microwave receivers can be installed outside the radiation shield, connected through oversized low-loss transmission lines of about $20 \mathrm{~m}$ length in order to allow accessibility during magnet operation. To protect them from ECRH stray radiation adapted filters must be installed.

Only a limited set of seven diagnostics is considered to be mandatory for the first test operation of W7-X. Among these are the Dispersion Interferometer for the electron density measurement and the ECE radiometer. Several other diagnostics will use OP1.1 to prepare their operation at the beginning of the later physics program. Among these are three reflectometers located in the same port to allow for crosschecks: (1) A V-band Doppler reflectometer monitors edge density fluctuations and their poloidal propagation velocity. (2) An innovative fast steering Doppler reflectometry antenna is being prepared capable for a fast angular scan of the probing beam thereby scanning the $K$-spectrum of turbulence via the Bragg-condition and (3) a conventional 24-40 GHz poloidal correlation reflectometry plugin with 5 antennas characterizes density perturbations and their propagation near the separatrix.

\section{Dispersion Interferometry}

In W7-X a microwave interferometer would suffer from space restrictions which do not allow for large optics in opposite ports, as well as from densities in excess of $1 \cdot 10^{20} \mathrm{~m}^{-3}$ and long path-lengths in the plasma resulting in beam refraction and subsequent multiple reflections at vessel walls. The latter perturb the phase measurement introducing spurious signal paths. Instead, Dispersion Interferometry (DI) [6] based on a $10.6 \mu \mathrm{m} \mathrm{CO}_{2}$-laser ( $\mathrm{P}<20 \mathrm{~W}$ ) and its second harmonic will be used for density control and later density profile measurements. DI employs frequency doubler crystals (here $\mathrm{AgGaSe}_{2}$ : conversion efficiency $\sim 10^{-6}$ ) to create a second harmonic $5.3 \mu \mathrm{m}$ reference signal propagating along the same path such that a separate reference path is not required.

In $\mathrm{W} 7-\mathrm{X}$ the $10 \mu \mathrm{m} / 5 \mu \mathrm{m}$ beams of the single-channel $\mathrm{CO}_{2}$ DI interferometer are backreflected from a $50 \mathrm{~mm}$ diameter retroreflector outside the vessel thus passing the plasma twice. The sightline is shared with the Nd:YAG-laser for the Thomson scattering system to allow for cross-calibration. ZnSe and quartz windows have to be used respectively due to the different 
wavelength which makes it necessary that the sightlines are slightly $(\sim 3 \mathrm{deg})$ tilted with respect to each other with their crossing in the plasma. The retroreflector is fastened to the Thomson diagnostic support structure in the torus center, about $8 \mathrm{~m}$ apart from the launching mirror. This long overall distance made it necessary to develop a feedback controlled beam steering system to cope with thermal drifts during long pulses.

As the $10 \mu \mathrm{m} / 5 \mu \mathrm{m}$ laser beams return from the plasma the $10.6 \mu \mathrm{m}$ signal is also upconverted in a second doubler crystal and the phase measurement is performed using the $5.3 \mu \mathrm{m}$ signals. In order to separate launched and returning beams in the interferometer optics geometrically, both are $32 \mathrm{~mm}$ parallel shifted with respect to each other by the retroreflector. For an DI arrangement variations of the geometrical path length cancel as they may result from vibrations - e.g. due to turbo pumps and water flow in cooling pipes - or from long-term thermal drifts. The resulting phase excursions thus display dispersion in the plasma only and therefore widely remain $<2 \pi$. For $\mathrm{W} 7-\mathrm{X}$ with a double-pass path-length in the plasma of $1.44 \mathrm{~m}$ in total a phase change of $2 \pi$ corresponds to an average density of about $1.010^{20} \mathrm{~m}^{-3}$. Phase ambiguities modulo $\pi$ therefore can be avoided, tracking the phase over the whole discharge is not required and DI becomes an option for steady-state density control.

Although it has been shown that DI is intrinsically insensitive versus vibrations along the sightline $[7,8]$, vibrations perpendicular to the line-of sight may result in small phase perturbations as they modulate misalignment [9]. Therefore, the DI itself has been installed on a vertical, vibration isolated bench that consists of two granite plates held by a massive Alstructure on a vibration isolated concrete base plate. The retroreflector opposite the plasma is itself fastened to a smaller vibration isolated stone plate to decouple it from device vibrations also. Phase measurement uses a heterodyne modulation scheme applying an elasto-optical ZnSe modulator at modulation frequency $50 \mathrm{kHz}$ from a stabilized quartz oscillator which also determines the temporal resolution. The plasma induced phase shift is derived from the interference of the returning signals by direct digital sampling at $50 \mathrm{Ms} / \mathrm{s}$ followed by digital filtering, down conversion and a phase comparison fitting the data and comparing it with the 50 $\mathrm{kHz}$ modulation signal. This signal treatment is acieved by means of a Field Progammable Gate Array (FPGA) which provides data in real time.

Comparison with an conventional $10 \mu \mathrm{m} / 5 \mu \mathrm{m}\left(\mathrm{CO}_{2} / \mathrm{CO}\right)$ two-color interferometer set-up using defined vibrations induced by piezos showed that with the DI arrangement the sensitivity versus vibration induced phase excursions is reduced by about an order of magnitude [9]. The residual phase excursions corresponding to density variations of a few times $10^{17} \mathrm{~m}^{-3}$ (over the total path length of $1.44 \mathrm{~m}$ in the plasma) mainly result from spurious modulation of the laser source induced by components of the returning beam. More relevant for the diagnostics characteristic are slow phase drifts (timescale several seconds) resulting in a baseline drift of the density by up to $1.10^{18} \mathrm{~m}^{-3}$ which are determined by air convection modulating the refraction index. Under laboratory conditions they are mainly due to temperature fluctuations which are driven, e.g. by the heating of optical components [9]. Therefore as further improvement the temperature of selected optical elements in the interferometer setup is actively controlled and for long-pulse operation the whole setup including the laser beam path outside the vessel can be operated under dry nitrogen atmosphere. 


\section{Electron Cyclotron Emission Diagnostics - ECE}

The ECE measures the $2^{\text {nd }}$ harmonic $\mathrm{x}$-mode emission at $2.5 \mathrm{~T}$ operation by means of a heterodyne radiometer in the frequency band $126 \mathrm{GHz}$ to $162 \mathrm{GHz}$. Due to the large aspect ratio the cyclotron harmonics are well separated in frequency and do not overlap. As required for ECE profile measurements the toroidal position has been selected such that the local stellarator magnetic field increases along the line-of sight tokomak-like towards the inner side of the torus. At the plasma axis the magnetic field differs from the $2.5 \mathrm{~T}$ in the ECRH launching plane, thus the central plasma temperature can be measured without being masked by the strong $140 \mathrm{GHz}$ microwave stray radiation from the heating beams. The system will be fully operable for the first test plasmas to track the plasma evolution and measure the electron temperature profile, the latter provided that the plasma optical thickness is sufficient for an interpretation of the emission as blackbody radiation. Moreover, the ECRH power deposition profile and dynamic electron heat transport can be derived from heatwave analysis modulating the power of a gyrotron.

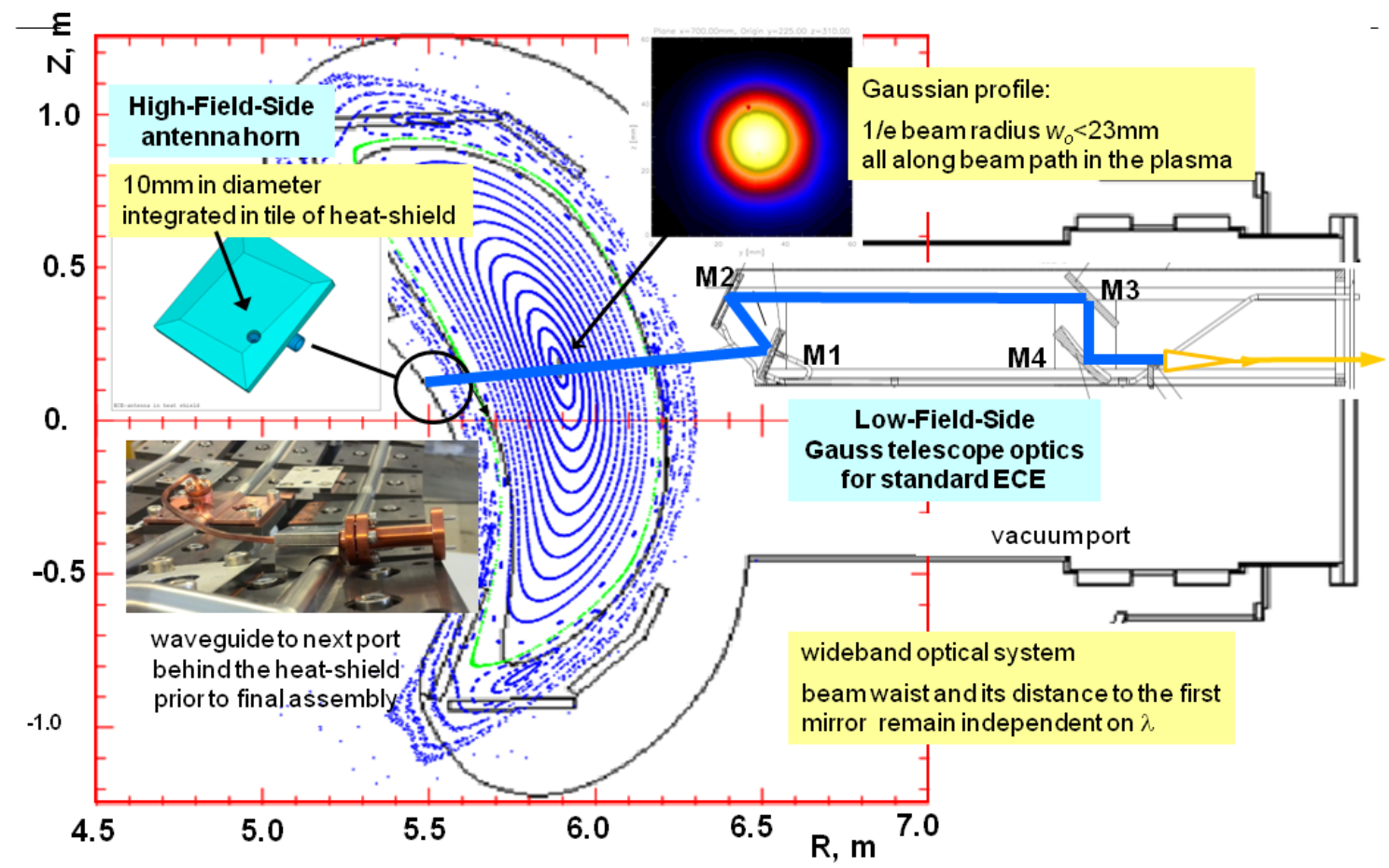

Fig. 1: Poloidal cross section at the ECE observation with the Low-Field Side Gauss plugin and the small High Field Side horn antenna indicated. Mirrors M2 and M4 of the Gauss optics are focussing, M1 and M3 are required for beam folding only.

Spatial resolution is maximized by minimizing the emitting volume for each frequency interval, i.e. a line-of-sight perpendicular to the flux surfaces and a slim Gaussian antenna characteristic focused at the plasma center with a 1/e beam radius $w_{0}$ remaining $w_{0}<23 \mathrm{~mm}$ all along the signal path through the plasma. The latter is achieved by intrinsically broadband Gaussian beam telescope optics, consisting of 2 elliptical and 2 plain stainless steel mirrors - the latter for beam folding and steering - and an adapted broadband horn antenna. This arrangement also keeps $\mathrm{Cu}$ components - microwave horn and fundamental mode waveguides - in the shadow of the mirrors far away from the plasma. For long-pulse operation the optics is prepared to be water cooled with cooling pipes integrated in the front mirrors. The vacuum barrier uses a 
$100 \mu \mathrm{m}$ thick Viton-sealed mica sheet with $4 \mathrm{~mm}$ aperture as broadband vacuum window. A 28 $\mathrm{mm}$ diameter oversized circular transmission line with overall length $\sim 22 \mathrm{~m}$ guides the $2 \mathrm{~mm}$ microwave radiation to the 32-channel ECE radiometer outside the experiment hall. In front of the radiometer the frequency band of stray radiation from the various gyrotrons (139.9 to 140.4 $\mathrm{GHz}$ ) is cut out of the spectrum by a waveguide Bragg reflection notch filter with depth $>40 \mathrm{~dB}$ (this value being limited by the available measurement equipment) [10] showing steep edges and an insertion loss of only 4 to $7 \mathrm{~dB}$ outside this frequency band.

The radiometer [11] uses a single broadband mixer for down conversion to an intermediate frequency range centred around $18 \mathrm{GHz}$ and subsequent $2-18 \mathrm{GHz}$ and $18-40 \mathrm{GHz}$ filterbanks, 16 channels each. The bandwidth of the individual filters $(0.25-1.4 \mathrm{GHz})$ corresponding to a radial resolution between 0.5 to $1 \mathrm{~cm}$ is adapted to the radial resolution of the ECE emission, as calculated from the optical depth at the expected plasma conditions [12]. For comparison the local plasma cross section along this sightline is $\sim 50 \mathrm{~cm}$, i.e the flux surfaces are compressed by about a factor of two with respect to a circular cross section with average minor radius a $\sim 50 \mathrm{~cm}$. The detector diode output is launched and filtered via differential amplifiers to the DAQ with maximum $2 \mathrm{Ms} / \mathrm{s}$ which allows to study fast mesoscale events also.

For higher spectral resolution a zoom IF-device [13] is available in parallel to the standard filterbank which allows the selection of any suitable frequency range of the spectrum by the aid of a tunable second local oscillator. In magnetic radial coordinates the selected frequency span of $4 \mathrm{GHz}$ covered with 16 channels corresponds to a radial range of $\Delta r \sim 6 \mathrm{~cm}$ at the High-Field Side (HFS) or $\sim 15 \mathrm{~cm}$ at the Low Field Side (LFS), respectively. This zoom device is particularly dedicated to perturbation experiments such as ELM studies or heatwave experiments from which the ECRH power deposition can be determined also.

For an overall absolute calibration of the diagnostic a second identical Gaussian optical system is provided as a twin outside the torus including identical waveguide components, mica window and a geometrically identical transmission line, however with a hot-cold calibration source chopping between $\mathrm{LN}_{2}$ temperature and room temperature in front of it. Laboratory measurements with this arrangement showed that the two optics can be relative calibrated with an accuracy of about $5 \%$ provided spurious reflections in the transmission path are carefully avoided.

A special feature of the ECE diagnostic is a second observation antenna with line-of-sight directed from the High-Field Side across the plasma towards the Gaussian optics enabling measurements of the emission from non-thermalized and current drive electrons at the same locations [14]. As for this wall location a heatload up to $400 \mathrm{~kW} / \mathrm{m}^{2}$ is expected a stainless steel microwave horn with a $10 \mathrm{~mm}$ aperture has been integrated in the high heatload graphite tiles and is thermally coupled to the water cooled CuCrZr heat sinks behind. The received microwave signals are guided to the next available port with rectangular overmoded waveguides in the shadow of the heat-shield. Outside the vessel this antenna is connected to the ECE radiometer via an oversized transmission line as well.

\section{Reflectometry}

During the first phase reflectometry measurements concentrate on the equatorial plane of the vertically elongated "bean shaped" poloidal cross section of W7-X where maximum turbulence amplitudes are expected from ballooning. The vertical plasma elongation resulting in 
minimum curvature of the cut-off surfaces and the steep density gradients with well localized reflection expected from an extrapolation of W7-AS results are considered to yield very good conditions for reflectometry. Two Doppler reflectometry antenna plugins and a conventional correlation reflectometry plugin will share this $100 \mathrm{~cm} \times 40 \mathrm{~cm}$ aperture port which allows for cross calibration (Fig. 3).

\subsection{Doppler Reflectometry}

Doppler reflectometry monitors edge density fluctuations and their poloidal propagation velocity from intensity and Doppler shift of the backscattered signal. For first exploration of W7-X the design strategy aims on a versatile Gauss antenna with circular horn output that allows both $\mathrm{x}$ - and o-mode with an extremely broadband design covering both V-band and Wband, 50-110 GHz. This gives access to the gradient region up to densities $1.510^{20} \mathrm{~m}^{-3}$. For Doppler reflectometry the line of sight is tilted with respect to the reflecting surface by $18^{\circ}$ by tilting the first mirror near the plasma poloidally. This selects turbulent structures with wavelength $0.5 \mathrm{~cm}<\Lambda_{\perp}<\mathrm{cm}$ via the Bragg condition $\Lambda_{-}=? \cdot \lambda_{\mu-\text { vave }} / \sin \theta$;

The optics is optimized to yield a maximum angular resolution as it is crucial to select the Doppler shifted backscattered signal from unshifted reflected signal components [15]. This is achieved by the plane wave fronts in the waist of a Gaussian beam which has a 1/e-folding width $w_{0}$ optimized with respect to the finite curvature of the reflecting layer i.e. this probed spot varies $w_{0} \sim \operatorname{sqrt}(\lambda)$ with $w_{0}(80 \mathrm{GHz})=2.4 \mathrm{~cm}$ at this specific location. The calculated spectral resolution of turbulent structures is $\Delta K / K=0.2-0.14$ depending on wavelength and configuration. Simultaneously an optics where the input waist varies $\sim \operatorname{sqrt}(\lambda)$ is intrinsically broadband as it has a constant Rayleigh-length. The optimized beam characteristics on the plasma side can be achieved with a suitable microwave horn and Gaussian transmission optics. However, the virtual beam waist within the horn inevitable shifts deeper into the horn for smaller wavelengths. In the case of a classical Gauss telescope this shift is transferred quadratically with the telescope magnification to a shift of the waist towards the plasma edge. This is just the opposite of what is desired if a density profile is taken into account where the cut-off for higher frequencies/smaller wavelengths is always deeper in the plasma. The latter can be overcome with a Gauss telescope optics with an odd number of focusing mirrors where the spot is deeper in the plasma for smaller wavelength as requested. The quadratic shift of the waist in the plasma with wavelength, however, remains. Therefore, an additional request to the horn design was that the wavelength dependent shift of the virtual waist in the horn should be minimized. Finally, mode purity of the transmission optics is limited by the aperture available for the first two stainless steel mirrors within the port. This is, however, reasonably balanced by the fact that the Gauss optics acts as a mode filter for higher order modes and suppresses side lobes which is crucial for Doppler reflectometry. The realized optics (Fig. 2) consists of 3 focussing and 3 plane stainless steel mirrors - the latter for beam folding in the port - and an electro-formed broadband Gaussian $\mathrm{Cu}$ horn with a length of $300 \mathrm{~mm}$. A second symmetrical optics views the same spot but with line of sight tilted by $-18^{\circ}$, i.e. in the poloidally opposite direction. This yields a compensation for possible geometrical asymmetries in the tilt angle as both red and blue shifted signal components can be measured but also allows for later density profile measurement with a bistatic antenna at the same location. An alternative modification for OP1.2 are two different 
values of tilt different angles which gives access to rapid changes in the turbulence $K$-spectrum by two Doppler reflectometry measurements simultaneously.

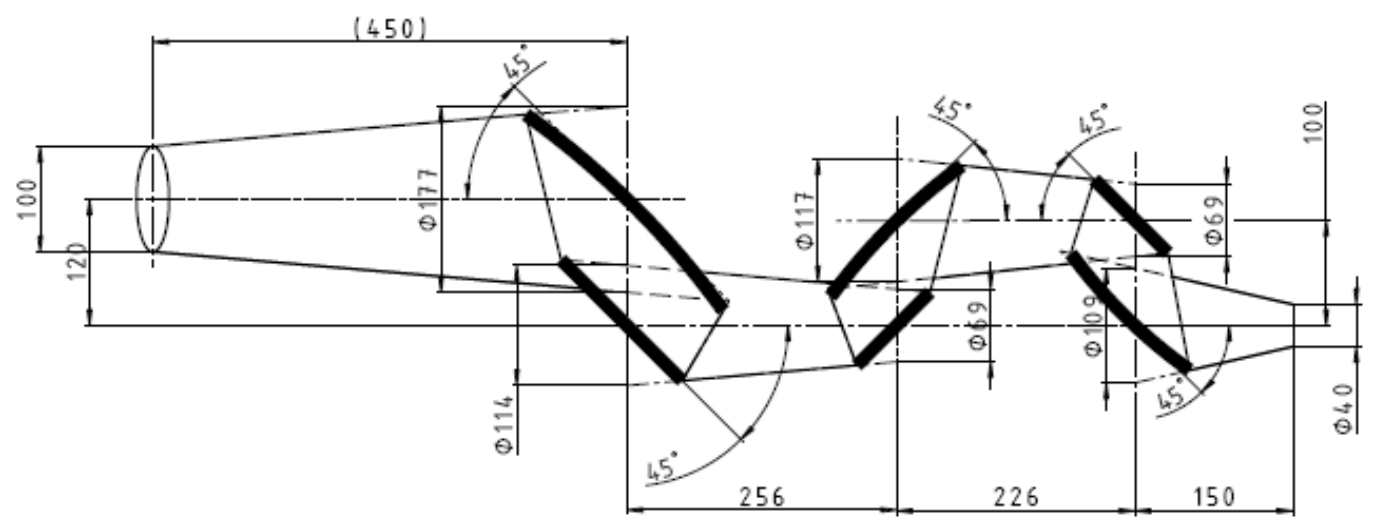

Fig. 2: Schematic top view of the broadband Gauss optics with 3 elliptical mirrors plus 3 plane mirrors for beam folding. The cut-off layer is on the left side, the broadband horn on the right in analogy to the next photograph below. The design cut-off layer with a beam width of $100 \mathrm{~mm}$ (left) is $450 \mathrm{~mm}$ apart from the first focussing mirror. For the magnetic standard configuration of $W 7-X$ this layer will be $4 \mathrm{~cm}$ behind the separatrix at $r / a=0.85$.

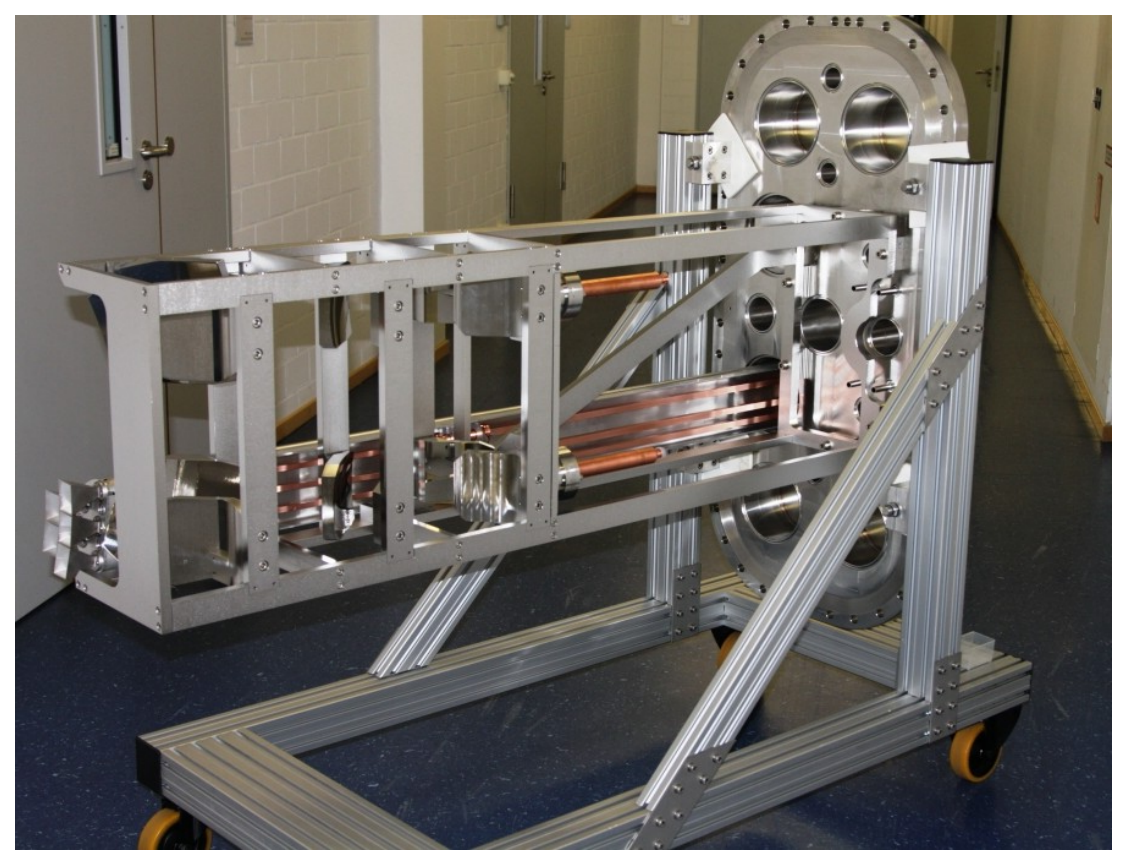

Fig. 3: Port closure with the plugins assembled for the Doppler reflectometry Gauss optics (towards the photographer) and for the conventional correlation reflectometry (lower rear, note the five microwave horns on the lower left side oriented towards the later plasma location). The plasma side of the plugins (left) will be slightly withdrawn with respect to the aperture of the port to the plasma vessel. The $1000 \mathrm{mmm} \times 400 \mathrm{~mm}$ port vacuum barrier (right) is situated about $2 \mathrm{~m}$ apart from the plasma resulting in the typical length of $W 7-X$ plugins of $\sim 1.8 \mathrm{~m}$. The frame of the Gauss optics is prepared to carry a cooled heat shield on the plasma side which later closes the port during long-pulse operation thus shielding the finally 9 diagnostics which will be integrated to this particular plugin during long pulse operation. Note the already prepared daughter flanges at the vacuum closure. The first mirrors of the reflectometry Gaus optics are already withdrawn with respect to the front side of this frame. 
Similar to ECE, the reflectometer transmitter will be outside the torus hall and connected by oversized circular transmission lines with single pass length $\sim 27 \mathrm{~m}$. For start-up a V-band (50-75 GHz) hopping reflectometer [16] is prepared for first Doppler reflectometry studies in the density gradient region $\left(3\right.$ to $\left.710^{19} \mathrm{~m}^{-3}\right)$.

In addition, a novel fast steering Doppler reflectometry antenna has been developed, capable of a fast angular scanning the probing beam, thereby scanning the K-spectrum of turbulence via the Bragg-condition [17]. This allows simultaneous access to shear-flow and $K$ spectrum of turbulence, i.e. the two quantities considered to be relevant for L-H transition physics via shear-flow decorrelation. This antenna consist of two sectoral horns with 32 stacked H-plane elements each and a phased feed array which allows variation of the beam tilt angle between \pm 20 deg by small frequency scans $\Delta f \sim 0.7 \mathrm{GHz}$ around 15 frequencies in the W-band, which define the reflecting layers. The plugin measures in direct neighbourhood of the Gauss optics plugin, enabling crosschecks.

\subsection{Correlation Reflectometry}

A conventional Ka-band, 24-40 GHz, poloidal correlation reflectometry plugin with one launching and 4 receiving antennas has been installed (Fig. 3) for measurements around the separatrix $\left(\mathrm{n}<210^{19} \mathrm{~m}^{-3}\right)$. It aims on characterization of density turbulence, edge mode activities and a measurement of the relatively low flow velocities in the velocity shear layer expected around the separatrix which can be derived via correlation techniques. This plugin is again attached to the same flange than the Doppler reflectometers, to allow for a cross-calibration of the measured propagation velocities.

\section{Further developments}

Planned developments for microwave and interferometer diagnostics are driven by technical challenges of the first experiment phases and the physics needs - namely the verification of the optimization criteria of an HELIAS type stellarator and the development of a high-density divertor-capable steady-state scenario with sufficiently low impurity confinement:

A 10 channel DI is being prepared to track the density profile shape in the core and study the necessity and success of deep fuelling as it may become necessary in an ECH heated stellarator where hollow density profiles are predicted [18]. In the physics exploration phase OP1.2 as a first step starting with 4 interferometer channels only continuous tracking of the separatrix (upstream) density for divertor control and a second core-channel density measurement for redundancy are requested. The W7-X stellarator configuration does not allow for large opposite ports. Instead, high-heatload Molybdenum retroreflectors have been developed which will be incorporated in the tiles of the heatshield [19].

For the expected high-density scenarios, with densities beyond $1.210^{20} \mathrm{~m}^{-3}$ where conventional X2 ECE is in cut-off continuous core temperature measurements need to be developed. They may use either Electron Bernstein wave Emission (EBE) with modeconversion observation geometries [20] or apply higher harmonics of ECE. Moreover, for densities $n<1.210^{20} \mathrm{~m}^{-3}$ standard ECE is considered as an option to investigate fast mesoscale events and turbulence in the plasma core.

Planned reflectometry extensions for OP1.2 include edge density profile measurements to track the upstream density and characterize the expected steep edge gradients. Conclusive 
turbulence studies under 3D plasma conditions presumably need access to further different locations of the torus. As a first approach a second identical V-band Doppler reflectometer is planned with optics at a toroidally shifted probing position with different poloidal cross section which also enables the identification of large scale flow structures.

The V-band Doppler reflectometer has received funding from the EUROfusion research and training programme 2014-2018.

\section{References}

[1] Klinger T. et al. 2013 Fus. Eng. Design 88461 (2013), S. Bosch et al. 2014 IEEE Transact Plasm. Sci. 42, 432

[2] Pedersen T. et al. 2014 21st EPS Conference on Plasma Physics Berlin O4.126

[3] König R. et al. this conference O4.4

[4] Dinklage A. et al. $201425^{\text {th }}$ IAEA FEC, St. Petersburg FIP/3-1

[5] Hirsch M. et al. 2013 Proc. Workshop on Fusion Rector Diag., 09.09.- 13.9.2013, AIP Conference Proceedings 1612 p39

[6] Bagryanski P. A. et al. 2006 Rev. Sci Instrum 77 053501, see T. Akiyama, I3.2, this conference.

[7] Kornejew P. et al. 2012 39th EPS Conference on Plasma Physics Stockholm P5037

[8] Akiyama T. et al. 2014 Rev. Sci. Instrum 85 11D301

[9] Kornejew, P. et al. Density measurements at fusion experiments: 2-color versus dispersion interferometers, to be published

[10] Wagner D. et al. 2011 J Infrared Milli Terahz Waves 32 1424-1433

[11] Fuchs Ch., Hartfuss H.-J. 2001 Rev. Sci. Instrum. 72383

[12] Schmuck S. et al. 2009 36th EPS Conference on Plasma Phys. Sofia ECA Vol.33E, P-4.212

[13] Fuchs Ch., Hartfuss H.-J. 2001 Fusion Eng. Des. 53 451-456

[14] Marushchenko et al. 2004 31st EPS Conference on Plasma Phys. London, ECA Vol.28G, P-1.204

[15] Hirsch M. et al. 2006 Nucl. Fusion 46 S853-S861

[16] Cupido L. et al. 2004 Rev. Sci. Instrum. 753865

[17] Rohmann P. et al. 2013 IEEE Int. Symposium on Phased Array Systems \& Technology, p 559

[18] Tanaka K. et al. 2010 Fus. Sci. Technol. 5870

[19] Peng X. B. et al. 2014 Fus. Eng and Design 89 318-323 and references therein

[20] Volpe F., Laqua H. P. 2003 Rev. Sci. Instrum. 74(3) 1409 